\title{
Article \\ Grain Yield, Nitrogen Use Efficiency and Antioxidant Enzymes of Rice under Different Fertilizer N Inputs and Planting Density
}

\author{
Wenxi Wang ${ }^{1,+}$, Congcong Shen ${ }^{1,2,+}$, Qin $\mathrm{Xu}^{3}{ }^{3}$, Sundus Zafar ${ }^{2}$, Bin Du ${ }^{1, *}$ and Danying Xing ${ }^{1, *}$ \\ 1 College of Agriculture, Yangtze University, Jingzhou 434025, China; 15927735542@163.com (W.W.); \\ shencc0805@163.com (C.S.) \\ 2 Shenzhen Branch, Guangdong Laboratory of Lingnan Modern Agriculture, Genome Analysis Laboratory of \\ the Ministry of Agriculture and Rural Affairs, Agricultural Genomics Institute at Shenzhen, \\ Chinese Academy of Agricultural Sciences, Shenzhen 518120, China; sundus_zafar@yahoo.com \\ 3 Institute of Crop Sciences, National Key Facility for Crop Gene Resources and Genetic Improvement, \\ Chinese Academy of Agricultural Sciences, Beijing 100081, China; xuqin@caas.cn \\ * Correspondence: xiaobin@stu.scau.edu.cn (B.D.); xingdy_2006@126.com (D.X.) \\ + These authors contributed equally to this work.
}

check for updates

Citation: Wang, W.; Shen, C.; Xu, Q.; Zafar, S.; Du, B.; Xing, D. Grain Yield, Nitrogen Use Efficiency and Antioxidant Enzymes of Rice under Different Fertilizer N Inputs and Planting Density. Agronomy 2022, 12, 430. https://doi.org/10.3390/ agronomy 12020430

Received: 19 November 2021

Accepted: 21 January 2022

Published: 9 February 2022

Publisher's Note: MDPI stays neutral with regard to jurisdictional claims in published maps and institutional affiliations.

Copyright: (ㅇ 2022 by the authors Licensee MDPI, Basel, Switzerland. This article is an open access article distributed under the terms and conditions of the Creative Commons Attribution (CC BY) license (https:// creativecommons.org/licenses/by/ $4.0 /)$.

\begin{abstract}
Nitrogen fertilizer and planting density are key factors affecting rice yield and nitrogen utilization efficiency. There is still a need to optimize nitrogen fertilizer and planting density management for high yield. We studied the effects of different quantities of nitrogen application $\left(\mathrm{N}_{0} 0 \mathrm{~kg} \mathrm{ha}{ }^{-1}, \mathrm{~N}_{1} 120 \mathrm{~kg} \mathrm{ha}^{-1}, \mathrm{~N}_{2} 180 \mathrm{~kg} \mathrm{ha}^{-1}\right)$ and planting density (low-density: 18.8 hills m$^{-2}$; high-density: 37.5 hills $\mathrm{m}^{-2}$ ) on rice yield, photosynthetic characteristics, antioxidant system, and nitrogen use efficiency. ANOVA results indicated that most tested traits were affected by environment, planting density, $\mathrm{N}$ application, and their interactions. Comparing the results of low-density planting, high-density planting increased the panicle number by $21.12 \%$ but decreased the grain number per panicle and yield by $3.97 \%$ and $22.48 \%$, respectively. Similarly, the superoxide dismutase (SOD), catalase (CAT), peroxidase (POD), and ascorbate peroxidase (APX) activities and PFPn (partial factor productivity of nitrogen) decreased by $8.20 \%, 6.99 \%, 16.41 \%, 14.28 \%$, and $5.73 \%$, respectively, while HIn ( $\mathrm{N}$ harvest index) increased by $1.32 \%$. Compared to no $\mathrm{N}$ application, $\mathrm{N}$ application treatments increased the panicle number, grain number per panicle, and yield by $9.92 \%, 17.64 \%$, and $37.83 \%$ in the $\mathrm{N}_{1}$ treatment; and increased by $17.15 \%, 29.09 \%$, and $128.94 \%$ in the $\mathrm{N}_{2}$ treatment, respectively. $\mathrm{N}$ application significantly increased net photosynthetic rates and enzyme activities of antioxidant antioxidases. Compared with $\mathrm{N}_{1}, \mathrm{~N}_{2}$ decreased REn (apparent recovery efficiency of N), AEn (agronomic N use efficiency), and PFPn by 8.98\%, 11.80\%, and 25.14\%, respectively, while, compared with $\mathrm{N}_{0}, \mathrm{~N}_{1}$ increased HIn by $8.50 \%$. It was observed that nitrogen fertilizer and planting density had an interaction effect on the net photosynthetic rate, antioxidant enzyme activities, and PFPn and HIn. Given a comprehensive consideration, it is best to apply nitrogen at a rate of

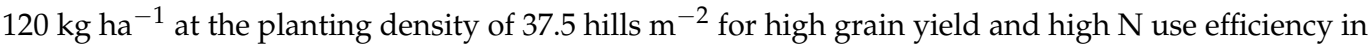
our experimental site.
\end{abstract}

Keywords: planting density; grain yield; nitrogen use efficiency; photosynthetic characteristics; antioxidant system

\section{Introduction}

Rice (Oryza sativa L.) is one of the most important food crops globally and the staple food of nearly half of the world's population, so it plays an important role in global food production and security [1]. An increase in rice yield will play an important role in future food production. The sustainable and healthy development of rice production and the steady growth of rice yield contribute to national food security [2]. With the acceleration 
of urbanization, the arable land area is decreasing, and the increase in rice yield must be obtained by improving productivity by increasing the yield per unit area [3,4].

Nitrogen $(\mathrm{N})$ is critical to the growth and development of rice and participates in many metabolic processes, such as protein hydrolysis and amino acids metabolism [3]. Nitrogen uptake is affected by various characteristics, fertilizer application, soil conditions, and environmental factors [5]. To meet the increasing demand for food in China, a large amount of nitrogen fertilizer is applied during agricultural production to obtain a greater yield [6]. However, excessive nitrogen fertilizer application has increased surface runoff and nitrification, resulting in $\mathrm{N}$ loss $[7,8]$. At present, the average recovery efficiency of nitrogen fertilizer is $33 \%$ at the field level [9]. Losses of $\mathrm{N}$ from the rice system would hamper the $\mathrm{N}$ use efficiency and could cause a series of environmental problems, including water eutrophication, soil hardening, acid rain, and the greenhouse effect [10,11]. In addition, it increases the sensitivity of crops to lodging and disease, and it limits the opportunities to increase rice yields [12]. Therefore, it is vital to improve crop $\mathrm{N}$ use efficiency to achieve a high yield [13].

The transplanting density of rice is the initial growth and development of a rice population, which can significantly affect the yield, population growth, and development of rice [14]. It is reported that increasing the transplanting density can increase the effective panicle number and yield of rice [15], and reducing transplanting density can increase the number of grains per spike and yield [16]. Huang et al. [17] found that increased density could compensate for yield loss caused by reduced N inputs. High transplanting density could increase the accumulation of dry matter in the early stage of growth and development [18], which might be attributed to the greater potential of crop canopies to capture solar radiation, water, and nutrients in the deeper soil layer [19]. Nevertheless, an excessive transplanting density of rice will reduce the distances between rows and between plants, and increase the length of the internode at the base of the stem, which will reduce the flexural strength of the stem and increase the risk of rice lodging [20]. An appropriate reduction of rice transplanting density can significantly improve the physical and chemical properties of rice stems and enhance their lodging resistance. However, it has little effect on the material accumulation in the middle and late stages, and even caused the reduction of dry matter accumulation in the middle and late stages of rice growth and development [21]. A slight increase of rice row spacing can significantly improve the canopy permeability, light interception ability, and light energy utilization efficiency of rice, leading to an increase in photosynthetic capacity and material accumulation capacity of rice leaves in the middle and later stages of growth and development [22]. As for the suitable transplanting density of rice, research results have been inconsistent to date. Generally speaking, planting rice close together will be more conducive to developing rice yield potential.

Appropriate rice transplanting density and a reasonable combination of nitrogen fertilizer application levels can increase the nitrogen fertilizer absorption and utilization of rice, affecting the growth efficiency and yield with an interacting effect [23,24]. From the perspective of protecting the environment, several researchers have increased the transplanting density and reduced the amount of nitrogen application, which improved the utilization rate of nitrogen fertilizer, and reduced the pollution of nitrogen fertilizer to the environment $[17,24-28]$. However, to achieve higher yields and lower pollution levels, there is still room for optimization in selecting planting density and nitrogen application rates. Previous studies have focused on yield increase and nitrogen use efficiency, while the effects of planting density and nitrogen application rate on rice physiology were rarely reported. Here, we investigated the effects of planting density and the nitrogen application rate on rice photosynthesis, nitrogen use efficiency, and yield in Jingzhou city, Hubei Province, China, from 2017 to 2019. 


\section{Material and Methods}

\subsection{Experimental Site Description}

We conducted experiments from 2017 to 2019 at the Yangtze University Farm, Jingzhou County $\left(30.33^{\circ} \mathrm{N}, 112.23^{\circ} \mathrm{E}, 36 \mathrm{~m}\right)$, Hubei Province, China. The area lies in the northern subtropical agricultural climate zone. The annual average temperature is $16.5^{\circ} \mathrm{C}$, the accumulated temperature $\geq 10{ }^{\circ} \mathrm{C}$ is $5094.9-5204.3^{\circ} \mathrm{C}$, the annual average precipitation is $1095 \mathrm{~mm}$, and the sunshine rate is $1718 \mathrm{~h}$. Meteorological data from 2017 to 2019 are shown in Figure 1. The soil type was sandy loam, and the soil characteristics are shown in Table 1. Chang-nong-geng 1 (CNG1) bred by Yangtze University was used as material in this study, which possesses excellent rice quality and high yield with a growth duration of 124-130 days from sowing to maturity.

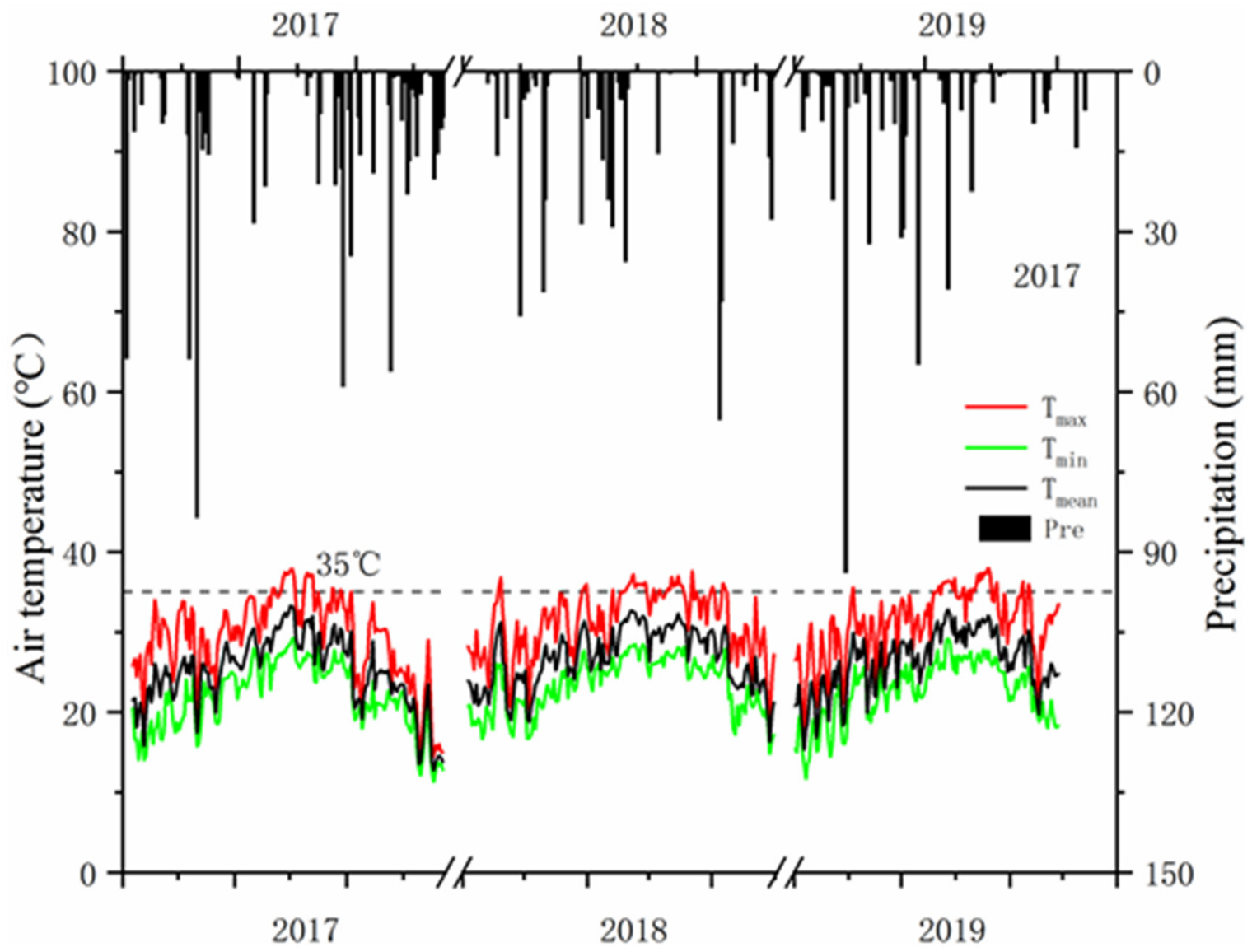

Figure 1. Meteorological data from 2017-2019. $T_{\max }, T_{\min }, T_{m e a n}$, and Pre are the maximum temperature, minimum temperature, average temperature, and precipitation, respectively.

Table 1. Average soil characteristics $(n=3)$ of experiment field composite topsoil samples $(0-20 \mathrm{~cm})$. The experiment was conducted at Yangtze University farm, Jingzhou County, Hubei Province, China (2017-2019).

\begin{tabular}{ccccccc}
\hline Year & $\begin{array}{c}\text { Total } \mathbf{~} \\
\left(\mathbf{g ~ k g}^{-\mathbf{1}}\right)\end{array}$ & $\begin{array}{c}\text { Available } \mathbf{~} \\
\left(\mathbf{m g ~ k g}^{-\mathbf{1}}\right)\end{array}$ & $\begin{array}{c}\text { Total } \mathbf{P} \\
\left(\mathbf{g ~ k g}^{-\mathbf{1}}\right)\end{array}$ & $\begin{array}{c}\text { Total K } \\
\left(\mathbf{g ~ k g}^{-\mathbf{1}}\right)\end{array}$ & $\begin{array}{c}\text { Organic Matter } \\
\left(\mathbf{g ~ k g}^{-\mathbf{1}}\right)\end{array}$ & $\mathbf{p H}$ \\
\hline 2017 & 2.24 & 83.94 & 0.57 & 3.49 & 21.61 & 6.80 \\
2018 & 2.27 & 87.16 & 0.53 & 3.55 & 21.41 & 6.70 \\
2019 & 2.25 & 86.88 & 0.59 & 3.67 & 21.39 & 6.70 \\
\hline
\end{tabular}

\subsection{Experimental Treatment and Design}

The experiment was conducted from 2017 to 2019 and laid out in a split-plot design with planting density as the main plot and nitrogen application as the subplot. Two planting 
densities and three nitrogen application rates were tested with three replicates, totaling 18 subplots $(4.5 \mathrm{~m} \times 6.5 \mathrm{~m})$ in the field. The planting densities were 37.5 and 18.8 hills $\mathrm{m}^{-2}$, representing hill spacings of $13.3 \times 20 \mathrm{~cm}$ and $26.7 \times 20 \mathrm{~cm}$, respectively. The nitrogen application rates were 0,120 , and $180 \mathrm{~kg} \mathrm{~N} \mathrm{ha}^{-1}$. Nitrogen in the form of urea was splitapplied with $40 \%$ as a basal fertilizer, $30 \%$ at mid-tillering, and $30 \%$ at flowering stages. $\mathrm{P}$ and $\mathrm{K}$ fertilizers were applied as a basal fertilizer $\left(\mathrm{kg} \mathrm{ha}^{-1}\right): \mathrm{P}_{2} \mathrm{O}_{5} 59$ and $\mathrm{K}_{2} \mathrm{O} 120$ were applied in the form of $\left(\mathrm{NH}_{4}\right)_{2} \mathrm{HPO}_{4}$ and $\mathrm{KCl}$, respectively.

Rice was sown on May 1 and transplanted on June 1 in 2017-2019. One or two seedlings were transplanted per hill in each experiment. Each main plot was surrounded by a $35 \mathrm{~cm}$ wide ridge covered with a plastic film. The plastic film was installed to a depth of $20 \mathrm{~cm}$ below the soil surface three days before transplanting. Weeds, pests, and diseases were intensively controlled to avoid yield loss.

\subsection{Measurement Items and Methods}

\subsubsection{Yield and Yield Components}

Grain yields were measured at maturity by taking $5 \mathrm{~m}^{2}$ plant samples at the center of each plot. Grains were separated from the rachis, filled and unfilled grains were separated, and the total weight of filled grains was determined. Filled grains were dried in an oven at $70{ }^{\circ} \mathrm{C}$ to a constant weight, and grain yield was calculated at $14 \%$ moisture content. Plant samples were taken to determine the yield components (panicle density, number of spikelets per panicle, 1000-grain weight, and seed setting rate) from 12 hills $\left(0.48 \mathrm{~m}^{2}\right)$ adjacent to the harvest area.

\subsubsection{Photosynthetic Rate}

The photosynthetic rate of the top fully-expanded leaves of the mainstem was determined at the mid-tillering, heading, and maturity stages. Five leaves were chosen for each treatment. A gas exchange analyzer (Li-6400, Li-COR Inc., Lincoln, NE, USA) was used to take the measurements between 9:30-11:00 a.m., when the active photosynthetic radiation above the canopy was $1200 \mathrm{mmol} \mathrm{m}^{-2} \mathrm{~s}^{-1}$.

\subsubsection{Antioxidase}

The fresh flag leaf samples at the panicle initiation stage were ground with liquid nitrogen to form homogenate, and $9 \mathrm{~mL}$ of $50 \mathrm{mM}$ sodium phosphate buffer (pH 7.8) was added to the homogenate and centrifuged at $8000 \mathrm{rpm}$ for $15 \mathrm{~min}$ at $4{ }^{\circ} \mathrm{C}$. The activities of superoxide dismutase (SOD) at $560 \mathrm{~nm}$, catalase (CAT) at $240 \mathrm{~nm}$, peroxidase (POD) at $470 \mathrm{~nm}$, and ascorbate peroxidase (APX) at $290 \mathrm{~nm}$ were determined according to the methods of Du et al. [29].

\subsubsection{Nitrogen Use Efficiency}

The grain and straw harvested at maturity were used for tissue $\mathrm{N}$ analysis after drying at $80{ }^{\circ} \mathrm{C}$ to a constant weight and weighed. $\mathrm{N}$ content in the grains and straw was determined with an automatic Kjeldahl Apparatus (FOSS-8400, Höganäs, Sweden). The apparent recovery efficiency of $\mathrm{N}\left(\mathrm{RE}_{\mathrm{N}}\right)$, agronomic $\mathrm{N}$ use efficiency $\left(\mathrm{AE}_{\mathrm{N}}\right)$, partial factor productivity of applied $\mathrm{N}(\mathrm{PFPn})$, physiological $\mathrm{N}$ use efficiency $\left(\mathrm{PE}_{\mathrm{N}}\right)$, internal $\mathrm{N}$ use efficiency $\left(\mathrm{IE}_{\mathrm{N}}\right)$, and $\mathrm{N}$ harvest index $\left(\mathrm{HI}_{\mathrm{N}}\right)$ were calculated according to the method of $\mathrm{Gu}$ et al. [30].

\subsection{Statistical Analyses}

Statistical data were analyzed using variance tests (ANOVA) in SPSS 21.0, and the differences between treatments were separated using the least significant difference (LSD) tests at $5 \%$ probability. The figure was drawn by Origin 9.1 (OriginLab Inc., Northampton, MA, USA). 


\section{Results}

\subsection{Effect of Nitrogen Application and Planting Density on Yield and Yield Components}

Table 2 shows summary statistics of the grain yield and its components under two density and three $\mathrm{N}$ applications across years. ANOVA results indicated that the differences among years and $\mathrm{N}$ applications, between densities, and variances due to interactions between any two factors (year, density, and $\mathrm{N}$ application) and among the three factors were significant or highly significant for most tested traits. Density had significant effects on the number of panicles, the number of grains per panicle, thousand-grain weight, and rice yield. Under different nitrogen treatments, the trend of panicle number and yield was $\mathrm{LD}<\mathrm{HD}$, though other traits show $\mathrm{HD}<\mathrm{LD}$. Compared with low-density planting, high-density planting increased the panicle number and yield by $21.12 \%$ and $22.48 \%$ and decreased the grain number per panicle by $3.97 \%$, respectively. Nitrogen application had a significant impact on the number of ears, grains per ear, thousand-grain weight, and rice yield. Under different density treatments with the trend of $\mathrm{N}_{0}<\mathrm{N}_{1}<\mathrm{N}_{2}$. Nitrogen had a significant impact on the seed setting rate with a trend of $\mathrm{N}_{2}<\mathrm{N}_{1}<\mathrm{N}_{0}$. Compared with no $\mathrm{N}$ application, the $\mathrm{N} 1$ treatment increased the panicle number, grain number per panicle, and yield by $9.92 \%, 17.64 \%$, and $37.83 \%$, respectively, while the N2 treatment increased the panicle number, grain number per panicle, and yield by $17.15 \%, 29.09 \%$, and $128.94 \%$, respectively. These results indicated that yield decreased with planting density while increased with $\mathrm{N}$ application.

Table 2. Effect of nitrogen application and planting density on yield and yield components. Different lowercase letters after the same column of values represented significant differences $(p<0.05)$ in the same year according to LSD. Values are the mean \pm standard error $(n=3)$. ns: denotes non-significance; ${ }^{*}, * *$ and ${ }^{* * *}$ : denote significance at $p<0.05,0.01$ and 0.001 , respectively.

\begin{tabular}{|c|c|c|c|c|c|c|c|}
\hline Year & Density & Nitrogen & $\begin{array}{l}\text { Panicle Number } \\
\qquad\left(\mathrm{m}^{2}\right)\end{array}$ & $\begin{array}{l}\text { Grain Number } \\
\text { per Panicle }\end{array}$ & $\begin{array}{c}\text { Seed Setting Rate } \\
(\%)\end{array}$ & $\begin{array}{c}\text { 1000-Grain Weight } \\
\text { (g) }\end{array}$ & $\begin{array}{c}\text { Grain Yield } \\
\mathrm{Kg} \mathrm{ha}^{-1}\end{array}$ \\
\hline \multirow{6}{*}{2017} & Low & 0 & $243.02 \pm 21.32 \mathrm{~d}$ & $107.08 \pm 6.07 c$ & $86.85 \pm 1.94 \mathrm{a}$ & $27.93 \pm 1.12 \mathrm{ab}$ & $6875.18 \pm 301.54 \mathrm{e}$ \\
\hline & Low & 120 & $277.58 \pm 10.59 \mathrm{~cd}$ & $125.52 \pm 7.33 \mathrm{ab}$ & $86.37 \pm 1.31 \mathrm{a}$ & $28.30 \pm 0.74 \mathrm{a}$ & $9399 \pm 513.06 c$ \\
\hline & Low & 180 & $309.40 \pm 38.32$ bc & $141.38 \pm 3.47 \mathrm{a}$ & $82.13 \pm 2.25 b$ & $28.33 \pm 0.74 \mathrm{a}$ & $12,020.45 \pm 672.57 \mathrm{a}$ \\
\hline & High & 0 & $312.99 \pm 15.69 \mathrm{abc}$ & $102.46 \pm 12.57 \mathrm{c}$ & $84.71 \pm 2.80 \mathrm{ab}$ & $26.48 \pm 0.87 b$ & $8313.39 \pm 338.08 \mathrm{~d}$ \\
\hline & High & 120 & $340.72 \pm 11.14 \mathrm{ab}$ & $117.22 \pm 9.62 \mathrm{bc}$ & $84.47 \pm 1.76 \mathrm{ab}$ & $26.98 \pm 1.11 \mathrm{ab}$ & $10,275.74 \pm 467.44 b$ \\
\hline & High & 180 & $355.27 \pm 20.42 \mathrm{a}$ & $129.74 \pm 12.72 \mathrm{ab}$ & $84.05 \pm 0.66 \mathrm{ab}$ & $27.79 \pm 0.24 \mathrm{ab}$ & $12,142.64 \pm 761.39 a$ \\
\hline \multirow{6}{*}{2018} & Low & 0 & $267.33 \pm 23.45 \mathrm{~d}$ & $103.46 \pm 4.96 c$ & $80.02 \pm 3.19 \mathrm{a}$ & $26.30 \pm 1.40 \mathrm{a}$ & $6760.89 \pm 293.81 \mathrm{~d}$ \\
\hline & Low & 120 & $305.34 \pm 11.65 c$ & $123.27 \pm 1.39 \mathrm{ab}$ & $76.46 \pm 3.75 \mathrm{ab}$ & $27.60 \pm 0.72 \mathrm{a}$ & $9907.6 \pm 381.05 \mathrm{~b}$ \\
\hline & Low & 180 & $340.34 \pm 23.67 \mathrm{~b}$ & $133.89 \pm 13.77 \mathrm{a}$ & $73.58 \pm 3.13 b$ & $27.97 \pm 1.66 \mathrm{a}$ & $11,944.88 \pm 776.25 \mathrm{a}$ \\
\hline & High & 0 & $344.29 \pm 17.26 \mathrm{~b}$ & $106.34 \pm 4.90 \mathrm{c}$ & $79.29 \pm 3.05 \mathrm{ab}$ & $27.09 \pm 0.37 \mathrm{a}$ & $9202.4 \pm 562.99$ c \\
\hline & High & 120 & $374.79 \pm 12.26 \mathrm{ab}$ & $120.82 \pm 3.88 b$ & $77.39 \pm 1.08 \mathrm{ab}$ & $27.46 \pm 0.32 \mathrm{a}$ & $11,777.75 \pm 525.56 \mathrm{a}$ \\
\hline & High & 180 & $390.79 \pm 22.46 \mathrm{a}$ & $125.31 \pm 1.60 \mathrm{ab}$ & $75.57 \pm 3.32 \mathrm{ab}$ & $26.71 \pm 0.64 \mathrm{a}$ & $12,085.52 \pm 573.21 \mathrm{a}$ \\
\hline \multirow{6}{*}{2019} & Low & 0 & $239.94 \pm 10.06 \mathrm{~d}$ & $96.59 \pm 7.13 \mathrm{~d}$ & $78.67 \pm 3.07 \mathrm{a}$ & $28.07 \pm 0.99 \mathrm{a}$ & $5890.52 \pm 346.88 \mathrm{f}$ \\
\hline & Low & 120 & $271.25 \pm 9.75 c$ & $125.41 \pm 6.55 \mathrm{ab}$ & $76.41 \pm 1.78 \mathrm{ab}$ & $27.42 \pm 0.92 \mathrm{ab}$ & $8335.34 \pm 517.67 \mathrm{~d}$ \\
\hline & Low & 180 & $305.33 \pm 24.31 b$ & $133.15 \pm 8.69 \mathrm{a}$ & $76.52 \pm 1.31 \mathrm{ab}$ & $28.24 \pm 0.36 \mathrm{a}$ & $10,792.49 \pm 361.78 b$ \\
\hline & High & 0 & $322.86 \pm 10.08 \mathrm{ab}$ & $100.18 \pm 7.62 \mathrm{~cd}$ & $76.46 \pm 2.25 \mathrm{ab}$ & $26.05 \pm 0.65 b$ & $7772.91 \pm 403.85 \mathrm{e}$ \\
\hline & High & 120 & $332.45 \pm 0.91 \mathrm{a}$ & $112.53 \pm 5.54 b c$ & $77.10 \pm 1.71 \mathrm{ab}$ & $27.72 \pm 0.51 \mathrm{ab}$ & $9581.52 \pm 447.51 \mathrm{c}$ \\
\hline & High & 180 & $326.05 \pm 11.57 \mathrm{ab}$ & $131.88 \pm 13.03 \mathrm{a}$ & $73.56 \pm 2.89 \mathrm{~b}$ & $28.06 \pm 1.82 \mathrm{a}$ & $11,692.59 \pm 420.98 \mathrm{a}$ \\
\hline \multicolumn{8}{|c|}{ ANOVA } \\
\hline \multicolumn{3}{|c|}{ Year $(Y)$} & $* * *$ & $* *$ & $* * *$ & * & $* * *$ \\
\hline \multicolumn{3}{|c|}{ Density (D) } & $* * *$ & * & ns & $* * *$ & $* * *$ \\
\hline \multicolumn{3}{|c|}{ Nitrogen $(\mathrm{N})$} & $* * *$ & $* * *$ & $* * *$ & $* * *$ & $* * *$ \\
\hline \multicolumn{3}{|c|}{$\mathrm{Y} \times \mathrm{D}$} & * & ns & ** & ** & $* * *$ \\
\hline \multicolumn{3}{|c|}{$\mathrm{Y} \times \mathrm{N}$} & ns & * & $* *$ & $* *$ & $* * *$ \\
\hline \multicolumn{3}{|c|}{$\mathrm{D} \times \mathrm{N}$} & $* * *$ & ** & $* * *$ & ns & $* * *$ \\
\hline \multicolumn{3}{|c|}{$\mathrm{Y} \times \mathrm{D} \times \mathrm{N}$} & $* * *$ & ns & $* * *$ & $* * *$ & * \\
\hline
\end{tabular}




\subsection{Effect of Nitrogen Application and Planting Density on Net Photosynthetic Rate}

Table 3 shows the summary statistics of the net photosynthetic rate at different growth stages under two density and three $\mathrm{N}$ applications over years. ANOVA results indicated that the differences among years and $\mathrm{N}$ applications, between densities, and variances due to interactions between any two factors (year, density, and $\mathrm{N}$ application) and among the three factors were significant or highly significant for most tested traits. With the maturity of rice, the net photosynthetic rate increased first and then decreased, showing $\mathrm{N}_{0}<\mathrm{N}_{2}<\mathrm{N}_{1}$, and the performance was the same for three years (Table 3). At low density, the net photosynthesis of high-nitrogen treatment was higher than that of low-nitrogen treatment, showing $\mathrm{N}_{0}<\mathrm{N}_{1}<\mathrm{N}_{2}$, and the trend was the same for three years. At high densities, net photosynthesis increased first and then decreased, consistent with the performance of rice reproductive stages in different years. Compared with no $\mathrm{N}$ application, $\mathrm{N}$ application increased net photosynthetic rates at the mid-tillering, heading, and maturity stages by $31.77 \%, 21.89 \%$, and $15.49 \%$, respectively. There was no significant difference in net photosynthetic rates between the $\mathrm{N}_{1}$ and $\mathrm{N}_{2}$ treatments. Under $\mathrm{N}_{0}$ and $\mathrm{N}_{1}$ treatments, overall, the net photosynthetic rate of low density was lower than that of high density, but the net photosynthesis rate under $\mathrm{N}_{1}$ treatment did not increase with the increase of density. Under $\mathrm{N}_{2}$ treatment, the net photosynthetic rate at low density was higher than that at high density. Compared with low-density planting, high-density planting led to decreased net photosynthetic rates at the mid-tillering and heading stages by $4.99 \%$ and $5.28 \%$, respectively. These results showed that increased planting density reduced the net photosynthetic rate and that the net photosynthetic rate increased to some extent with $\mathrm{N}$ application.

Table 3. Effect of nitrogen application and planting density on net photosynthetic rate ( $\mathrm{Pn})$ $\left(\mu \mathrm{M} \mathrm{CO}_{2} \mathrm{~m}^{-2} \mathrm{~s}^{-1}\right)$ at different growing stages. Different lowercase letters after the same column of values represented significant differences $(p<0.05)$ in the same year according to LSD. Values are the mean \pm standard error $(n=3)$. ns: denotes non-significance; ${ }^{*}, * *$ and ${ }^{* * *}$ : denote significance at $p<0.05,0.01$ and 0.001 , respectively.

\begin{tabular}{|c|c|c|c|c|c|}
\hline Year & Density & Nitrogen & $\begin{array}{l}\text { At Mid-Tillering } \\
\mu \mathrm{M} \mathrm{CO} \mathrm{C}_{2}^{-2} \mathrm{~s}^{-1}\end{array}$ & $\begin{array}{c}\text { At Heading } \\
\mu \mathrm{M} \mathrm{CO}_{2} \mathrm{~m}^{-2} \mathrm{~s}^{-1}\end{array}$ & $\begin{array}{c}\text { At Maturity } \\
\mu \mathrm{M} \mathrm{CO}_{2} \mathrm{~m}^{-2} \mathrm{~s}^{-1}\end{array}$ \\
\hline \multirow{6}{*}{2017} & Low & 0 & $18.80 \pm 0.35 \mathrm{~d}$ & $22.40 \pm 0.17 \mathrm{~cd}$ & $16.71 \pm 0.35 \mathrm{~d}$ \\
\hline & Low & 120 & $21.81 \pm 0.33 c$ & $25.16 \pm 0.64 \mathrm{ab}$ & $19.48 \pm 0.52 b$ \\
\hline & Low & 180 & $25.35 \pm 0.09 \mathrm{a}$ & $26.71 \pm 1.48 \mathrm{a}$ & $20.69 \pm 0.45 \mathrm{a}$ \\
\hline & High & 0 & $18.19 \pm 0.43 \mathrm{~d}$ & $21.43 \pm 0.35 \mathrm{~d}$ & $18.26 \pm 0.31 \mathrm{c}$ \\
\hline & High & 120 & $23.70 \pm 0.45 b$ & $25.27 \pm 1.03 b$ & $19.58 \pm 0.51 b$ \\
\hline & High & 180 & $21.53 \pm 0.96 \mathrm{c}$ & $23.62 \pm 0.53 c$ & $18.63 \pm 0.42 c$ \\
\hline \multirow{6}{*}{2018} & Low & 0 & $19.58 \pm 0.45 \mathrm{~d}$ & $23.81 \pm 0.22 \mathrm{c}$ & $18.37 \pm 0.24 b$ \\
\hline & Low & 120 & $23.70 \pm 0.35 b$ & $27.37 \pm 0.13 a$ & $20.62 \pm 0.75 \mathrm{a}$ \\
\hline & Low & 180 & $26.00 \pm 0.71 \mathrm{a}$ & $27.66 \pm 0.45 a$ & $21.58 \pm 0.25 \mathrm{a}$ \\
\hline & High & 0 & $19.31 \pm 0.29 \mathrm{~d}$ & $21.85 \pm 0.67 c$ & $19.08 \pm 0.26 b$ \\
\hline & High & 120 & $24.56 \pm 0.19 b$ & $25.59 \pm 0.71 b$ & $20.62 \pm 1.23 \mathrm{a}$ \\
\hline & High & 180 & $21.93 \pm 0.83 c$ & $26.00 \pm 0.64 b$ & $18.97 \pm 0.64 b$ \\
\hline \multirow{6}{*}{2019} & Low & 0 & $18.72 \pm 0.54 \mathrm{e}$ & $22.42 \pm 0.31 \mathrm{c}$ & $17.10 \pm 0.62 c$ \\
\hline & Low & 120 & $22.58 \pm 0.21 \mathrm{c}$ & $25.00 \pm 0.50 \mathrm{ab}$ & $19.22 \pm 0.50 \mathrm{ab}$ \\
\hline & Low & 180 & $25.30 \pm 0.64 a$ & $25.80 \pm 0.56 \mathrm{a}$ & $20.10 \pm 0.85 \mathrm{a}$ \\
\hline & High & 0 & $17.76 \pm 0.47 \mathrm{e}$ & $20.98 \pm 0.63 \mathrm{~d}$ & $18.70 \pm 0.14 b$ \\
\hline & High & 120 & $23.67 \pm 0.71 b$ & $25.35 \pm 0.75 \mathrm{ab}$ & $19.28 \pm 0.13 \mathrm{ab}$ \\
\hline & High & 180 & $21.18 \pm 0.76 \mathrm{~d}$ & $24.24 \pm 1.23 \mathrm{~b}$ & $18.68 \pm 0.38 b$ \\
\hline \multicolumn{6}{|c|}{ ANOVA } \\
\hline & Year (Y) & & $* * *$ & $* * *$ & $* * *$ \\
\hline & Density (D) & & $* * *$ & $* * *$ & * \\
\hline & Nitrogen (N) & & $* * *$ & $* * *$ & $* * *$ \\
\hline & $\mathrm{Y} \times \mathrm{D}$ & & $* *$ & $* *$ & ** \\
\hline & $\mathrm{Y} \times \mathrm{N}$ & & ** & ns & ns \\
\hline & $\mathrm{D} \times \mathrm{N}$ & & $* * *$ & $* * *$ & $* * *$ \\
\hline & $\mathrm{Y} \times \mathrm{D} \times \mathrm{N}$ & & * & $* * *$ & ** \\
\hline
\end{tabular}




\subsection{Effect of Nitrogen Application and Planting Density on Antioxidant Enzyme Activities}

Table 4 shows the summary statistics of antioxidant enzyme activities under two density and three $\mathrm{N}$ applications across years. ANOVA results indicated that the differences among years and $\mathrm{N}$ applications, between densities, and variances due to interactions between any two factors (year, density, and $\mathrm{N}$ application) and among the three factors were significant or highly significant for most antioxidant enzyme activities. Under lowdensity treatment, the antioxidant enzyme activity increased with an increase of nitrogen application at $\mathrm{N}_{2}>\mathrm{N}_{1}>\mathrm{N}_{0}$, and the performance was consistent over three years. Under the high-density treatment, with the increase of nitrogen, the antioxidant enzyme activity first increased then decreased, showing $\mathrm{N}_{1}>\mathrm{N}_{2}>\mathrm{N}_{0}$, but higher than the N0 treatment. Compared with no $\mathrm{N}$ application, SOD, CAT, POD, and APX activities increased by $19.51 \%$, $17.39 \%, 73.77 \%$, and $38.54 \%$ under the $\mathrm{N}_{1}$ treatment, and increased by $19.37 \%, 15.93 \%$, $68.53 \%$, and $44.12 \%$ under the $\mathrm{N}_{2}$ treatment, respectively. Under the treatment of nitrogen application, high density increased the activity of antioxidant enzymes. Compared with low-density planting, high-density planting led to decreased SOD, CAT, POD, and APX activities by $8.20 \%, 6.99 \%, 16.41 \%$, and $14.28 \%$, respectively. The results showed that excessive planting density reduced the activity of antioxidant enzymes, but that activity of antioxidant enzymes increased with $\mathrm{N}$ application.

Table 4. Effect of nitrogen application and planting density on antioxidant enzyme activities $\left(\mathrm{U} \min ^{-1} \mathrm{~g}^{-1} \mathrm{FW}\right.$ ). Different lowercase letters after the same column of values represented significant differences $(p<0.05)$ in the same year according to LSD. Values are the mean \pm standard error $(n=3)$. ns: denotes non-significance; ${ }^{*}$, and ${ }^{* * *}$ : denote significance at $p<0.05$, and 0.001 , respectively.

\begin{tabular}{|c|c|c|c|c|c|c|}
\hline Year & Density & Nitrogen & $\begin{array}{c}\text { SOD } \\
\mathrm{U} \mathrm{min}^{-1} \mathrm{~g}^{-1} \mathrm{FW}\end{array}$ & $\begin{array}{c}\text { POD } \\
\mathrm{U} \mathrm{min}^{-1} \mathrm{~g}^{-1} \mathrm{FW}\end{array}$ & $\begin{array}{c}\text { CAT } \\
\mathrm{U} \mathrm{min}^{-1} \mathrm{~g}^{-1} \mathrm{FW}\end{array}$ & $\begin{array}{c}\mathrm{APX} \\
\mathrm{U} \min ^{-1} \mathrm{~g}^{-1} \mathrm{FW}\end{array}$ \\
\hline \multirow{6}{*}{2017} & Low & 0 & $337.55 \pm 6.80 \mathrm{e}$ & $416.93 \pm 11.28 b$ & $176.41 \pm 5.10 \mathrm{e}$ & $134.18 \pm 2.06 \mathrm{~d}$ \\
\hline & Low & 120 & $407.23 \pm 2.77 b$ & $485.88 \pm 16.63 \mathrm{a}$ & $314.50 \pm 12.97 \mathrm{~b}$ & $181.27 \pm 3.74 b$ \\
\hline & Low & 180 & $430.91 \pm 10.97 a$ & $486.14 \pm 18.52 \mathrm{a}$ & $338.41 \pm 7.94 \mathrm{a}$ & $208.15 \pm 14.24 \mathrm{a}$ \\
\hline & High & 0 & $331.01 \pm 5.02 \mathrm{e}$ & $395.37 \pm 6.48 b$ & $166.36 \pm 7.51 \mathrm{e}$ & $118.09 \pm 2.31 \mathrm{e}$ \\
\hline & High & 120 & $393.13 \pm 7.67 c$ & $472.54 \pm 1.86 \mathrm{a}$ & $288.42 \pm 11.70 c$ & $167.54 \pm 4.93 c$ \\
\hline & High & 180 & $373.42 \pm 2.61 \mathrm{~d}$ & $437.22 \pm 9.05 b$ & $240.77 \pm 5.77 \mathrm{~d}$ & $158.87 \pm 3.91 \mathrm{c}$ \\
\hline \multirow{6}{*}{2018} & Low & 0 & $350.38 \pm 11.43 c$ & $416.42 \pm 8.76 c$ & $186.02 \pm 7.23 \mathrm{e}$ & $134.19 \pm 2.69 \mathrm{e}$ \\
\hline & Low & 120 & $409.91 \pm 9.36 \mathrm{a}$ & $471.00 \pm 24.28 \mathrm{~b}$ & $314.12 \pm 7.69 \mathrm{~b}$ & $184.76 \pm 6.25 b$ \\
\hline & Low & 180 & $425.43 \pm 7.78 \mathrm{a}$ & $510.87 \pm 1.98 \mathrm{a}$ & $338.84 \pm 15.02 \mathrm{a}$ & $205.86 \pm 4.81 \mathrm{a}$ \\
\hline & High & 0 & $322.17 \pm 6.75 \mathrm{~d}$ & $383.79 \pm 10.7 \mathrm{~d}$ & $165.13 \pm 8.43 \mathrm{f}$ & $120.30 \pm 3.92 \mathrm{f}$ \\
\hline & High & 120 & $386.21 \pm 2.93 \mathrm{~b}$ & $458.7 \pm 4.45 \mathrm{~b}$ & $288.11 \pm 13.53 c$ & $169.19 \pm 2.22 c$ \\
\hline & High & 180 & $364.55 \pm 16.58 c$ & $431.3 \pm 13.53 c$ & $251.80 \pm 10.22 \mathrm{~d}$ & $154.81 \pm 2.52 \mathrm{~d}$ \\
\hline \multirow{6}{*}{2019} & Low & 0 & $335.69 \pm 8.6 \mathrm{~d}$ & $413.35 \pm 17.65 \mathrm{~b}$ & $177.93 \pm 8.90 \mathrm{e}$ & $134.05 \pm 0.44 \mathrm{~d}$ \\
\hline & Low & 120 & $410.59 \pm 19.91 b$ & $467.11 \pm 3.39 \mathrm{a}$ & $317.27 \pm 6.04 b$ & $181.51 \pm 5.78 b$ \\
\hline & Low & 180 & $438.80 \pm 16.01 \mathrm{a}$ & $488.43 \pm 19.31 \mathrm{a}$ & $340.43 \pm 9.88 \mathrm{a}$ & $202.93 \pm 2.98 \mathrm{a}$ \\
\hline & High & 0 & $330.41 \pm 4.64 \mathrm{~d}$ & $380.68 \pm 8.87 c$ & $167.48 \pm 5.37 \mathrm{e}$ & $119.68 \pm 2.41 \mathrm{e}$ \\
\hline & High & 120 & $391.74 \pm 15.01 \mathrm{~b}$ & $469.87 \pm 12.01 \mathrm{a}$ & $283.64 \pm 5.73 c$ & $169.30 \pm 4.30 \mathrm{c}$ \\
\hline & High & 180 & $362.91 \pm 17.22 \mathrm{c}$ & $435.98 \pm 17.84 \mathrm{~b}$ & $241.31 \pm 4.39 \mathrm{~d}$ & $165.40 \pm 4.09 c$ \\
\hline \multicolumn{7}{|c|}{ ANOVA } \\
\hline \multicolumn{3}{|c|}{ Year (Y) } & $\mathrm{ns}$ & * & * & $\mathrm{ns}$ \\
\hline \multicolumn{3}{|c|}{ Density (D) } & $* * *$ & $* * *$ & $* * *$ & $* * *$ \\
\hline \multicolumn{3}{|c|}{ Nitrogen (N) } & $* * *$ & $* * *$ & $* * *$ & $* * *$ \\
\hline \multicolumn{3}{|c|}{$\mathrm{Y} \times \mathrm{D}$} & ns & * & ns & * \\
\hline \multicolumn{3}{|c|}{$\mathrm{Y} \times \mathrm{N}$} & * & $* * *$ & ns & ns \\
\hline \multicolumn{3}{|c|}{$\mathrm{D} \times \mathrm{N}$} & $* * *$ & $* * *$ & $* * *$ & $* * *$ \\
\hline \multicolumn{3}{|c|}{$\mathrm{Y} \times \mathrm{D} \times \mathrm{N}$} & * & * & $* * *$ & ns \\
\hline
\end{tabular}

\subsection{Effect of Nitrogen Application and Planting Density on N Use Efficiency}

Table 5 shows summary statistics of $\mathrm{N}$ use efficiency related traits under two density and three $\mathrm{N}$ applications over years. ANOVA results indicated that the differences among 
years and $\mathrm{N}$ applications between densities were significant or highly significant for most of $\mathrm{N}$ use efficiency related traits, while variances due to interactions between any two factors (year, density, and $\mathrm{N}$ application) and among the three factors were insignificant for most of $\mathrm{N}$ use efficiency related traits. Nitrogen application and planting density significantly affected rice REn, PFPn, IEn, and HIn. The interaction of nitrogen application by planting density significantly affected PFPn, Pen, and HIn across three years (Table 5). The impact on PFPn and HIn is significant. Compared with low-density planting, high-density planting led to a decrease in PFPn by $5.73 \%$, and an increase in HIn by $1.32 \%$. Compared with the $\mathrm{N}_{1}$ treatment, the $\mathrm{N}_{2}$ treatment decreased REn, AEn, and PFPn by $8.98 \%, 11.80 \%$, and $25.14 \%$, respectively. Compared with the $\mathrm{N}_{0}$ treatment, the $\mathrm{N}_{1}$ treatment increased HIn by $8.50 \%$. There was no difference in HIn between $\mathrm{N}_{0}$ and $\mathrm{N}_{2}$. Increasing $\mathrm{N}$ application rate had no significant effect on PFPn in low-density planting but significantly decreased PFPn by $11.34 \%$ in high-density planting. HIn ranked as $\mathrm{N}_{1}>\mathrm{N}_{2}>\mathrm{N}_{0}$ in low planting density and $\mathrm{N}_{1}>\mathrm{N}_{0}>\mathrm{N}_{2}$ in high-density planting. The above results explained that excessive $\mathrm{N}$ application and high planting density reduced the $\mathrm{N}$ use efficiency, especially when both were excessive.

Table 5. Effect of nitrogen application and planting density on $\mathrm{N}$ use efficiency. Different lowercase letters after the same column of values represented significant differences $(p<0.05)$ in the same year according to LSD. Values are the mean \pm standard error $(n=3)$. ns: denotes non-significance; ${ }^{*}, * *$ and ${ }^{* * *}$ : denote significance at $p<0.05,0.01$ and 0.001 , respectively.

\begin{tabular}{|c|c|c|c|c|c|c|c|c|}
\hline Year & Density & Nitrogen & $\begin{array}{l}\text { REn } \\
(\%)\end{array}$ & $\underset{\left(\mathrm{kg} \mathrm{kg}^{-1}\right)}{\mathrm{AEn}}$ & $\begin{array}{c}\text { PFPn } \\
\left(\mathrm{kg} \mathrm{kg}^{-1}\right)\end{array}$ & $\begin{array}{c}\text { PEn } \\
\left(\mathrm{kg} \mathrm{kg}^{-1}\right)\end{array}$ & $\begin{array}{c}\text { IEN } \\
\left(\mathrm{kg} \mathrm{kg}^{-1}\right)\end{array}$ & $\begin{array}{l}\text { HIn } \\
(\%)\end{array}$ \\
\hline \multirow{6}{*}{2017} & Low & 0 & & & & & & $62.50 \pm 2.04 \mathrm{~d}$ \\
\hline & Low & 120 & $32.36 \pm 2.86 \mathrm{a}$ & $28.41 \pm 2.44 \mathrm{a}$ & $65.18 \pm 1.57 \mathrm{a}$ & $88.40 \pm 12.24 \mathrm{a}$ & $101.73 \pm 6.66 \mathrm{a}$ & $68.57 \pm 0.32 b$ \\
\hline & Low & 180 & $30.90 \pm 1.67 \mathrm{a}$ & $25.76 \pm 3.33 \mathrm{a}$ & $50.27 \pm 4.03 b$ & $83.67 \pm 12.98 \mathrm{a}$ & $96.50 \pm 8.39 \mathrm{a}$ & $65.90 \pm 0.86 c$ \\
\hline & High & 0 & & & & & & $63.29 \pm 1.00 \mathrm{~d}$ \\
\hline & High & 120 & $34.38 \pm 1.71 \mathrm{a}$ & $26.54 \pm 5.68 \mathrm{a}$ & $66.77 \pm 3.41 \mathrm{a}$ & $76.78 \pm 12.42 \mathrm{a}$ & $98.21 \pm 3.33 \mathrm{a}$ & $69.21 \pm 0.42 \mathrm{a}$ \\
\hline & High & 180 & $30.92 \pm 1.76 \mathrm{a}$ & $22.92 \pm 3.23 \mathrm{a}$ & $49.74 \pm 1.82 b$ & $73.90 \pm 6.11 \mathrm{a}$ & $93.30 \pm 1.54 \mathrm{a}$ & $61.55 \pm 1.86 \mathrm{~d}$ \\
\hline \multirow{6}{*}{2018} & Low & 0 & & & & & & $63.11 \pm 1.90 \mathrm{bc}$ \\
\hline & Low & 120 & $35.16 \pm 0.80 \mathrm{a}$ & $27.04 \pm 3.53 \mathrm{a}$ & $61.55 \pm 3.48 b$ & $76.81 \pm 8.40 \mathrm{a}$ & $90.23 \pm 4.48 \mathrm{a}$ & $67.38 \pm 1.85 \mathrm{a}$ \\
\hline & Low & 180 & $30.62 \pm 2.21 \mathrm{a}$ & $23.82 \pm 4.82 \mathrm{a}$ & $46.82 \pm 4.44 \mathrm{c}$ & $78.53 \pm 19.28 \mathrm{a}$ & $89.23 \pm 11.60 \mathrm{a}$ & $64.63 \pm 1.16 b$ \\
\hline & High & 0 & & & & & & $64.00 \pm 0.53 b$ \\
\hline & High & 120 & $34.83 \pm 3.18 \mathrm{a}$ & $28.59 \pm 8.22 \mathrm{a}$ & $70.10 \pm 4.89 \mathrm{a}$ & $84.12 \pm 33.00 \mathrm{a}$ & $98.78 \pm 9.74 \mathrm{a}$ & $68.89 \pm 0.35 a$ \\
\hline & High & 180 & $33.45 \pm 2.47 \mathrm{a}$ & $20.40 \pm 3.12 \mathrm{a}$ & $48.08 \pm 3.39 c$ & $60.78 \pm 4.90 \mathrm{a}$ & $83.41 \pm 4.78 \mathrm{a}$ & $61.36 \pm 1.36 c$ \\
\hline \multirow{6}{*}{2019} & Low & 0 & & & & & & $63.62 \pm 1.39 c$ \\
\hline & Low & 120 & $31.57 \pm 4.20 \mathrm{ab}$ & $25.87 \pm 7.10 \mathrm{a}$ & $57.59 \pm 5.46 \mathrm{a}$ & $84.53 \pm 32.85 \mathrm{a}$ & $90.68 \pm 9.86 a$ & $68.46 \pm 1.50 \mathrm{a}$ \\
\hline & Low & 180 & $30.03 \pm 1.74 \mathrm{ab}$ & $25.70 \pm 2.16 \mathrm{a}$ & $46.84 \pm 0.69 \mathrm{a}$ & $85.57 \pm 4.37 \mathrm{a}$ & $91.29 \pm 4.08 \mathrm{a}$ & $66.17 \pm 2.10 b$ \\
\hline & High & 0 & & & & & & $63.94 \pm 0.67 \mathrm{bc}$ \\
\hline & High & 120 & $34.68 \pm 2.00 \mathrm{a}$ & $25.6 \pm 2.85 \mathrm{a}$ & $65.04 \pm 5.43 \mathrm{a}$ & $73.75 \pm 5.70 \mathrm{a}$ & $94.61 \pm 7.76 \mathrm{a}$ & $70.29 \pm 0.65 a$ \\
\hline & High & 180 & $28.84 \pm 1.22 \mathrm{~b}$ & $21.08 \pm 1.91 \mathrm{a}$ & $47.38 \pm 3.43 a$ & $73.36 \pm 9.51 \mathrm{a}$ & $92.06 \pm 9.34 \mathrm{a}$ & $59.99 \pm 0.52 \mathrm{~d}$ \\
\hline \multicolumn{9}{|c|}{ ANOVA } \\
\hline & Year (Y) & & $* * *$ & ns & $* *$ & ns & $* * *$ & ** \\
\hline & Density (D) & & ns & ns & $* * *$ & $* *$ & ns & $* *$ \\
\hline & Nitrogen $(\mathrm{N})$ & & $* * *$ & $* * *$ & $* * *$ & * & $* * *$ & $* * *$ \\
\hline & $\mathrm{Y} \times \mathrm{D}$ & & $\mathrm{ns}$ & ns & $* *$ & $\mathrm{~ns}$ & ns & * \\
\hline & $\mathrm{Y} \times \mathrm{N}$ & & $\mathrm{ns}$ & $\mathrm{ns}$ & ns & $\mathrm{ns}$ & * & $* * *$ \\
\hline & $\mathrm{D} \times \mathrm{N}$ & & ns & ns & $* * *$ & ns & ns & $* * *$ \\
\hline & $\mathrm{Y} \times \mathrm{D} \times \mathrm{N}$ & & $* * *$ & ns & * & ns & $* * *$ & ns \\
\hline
\end{tabular}

\section{Discussion}

The nitrogen fertilizer application rate, transplanting density, and their interaction were important factors affecting rice yield and nitrogen use efficiency [28-30]. To provide more data on the management of nitrogen fertilizer and transplanting density in rice production, we studied the effects of nitrogen fertilizer application, transplanting density, and their interaction on yield and its components, photosynthesis, antioxidant enzyme 
activities, and nitrogen use efficiency. We found that increasing the transplanting density increased the effective panicle number and decreased the grain number per panicle and yield, and these findings are consistent with Berhanu et al. [16]. Higher transplanting density can guarantee the initial population number of rice, such as the number of tillers, but an excessive number of tillers will reduce the rate of panicle formation, resulting in waste of carbohydrates and water accumulation in ineffective tillers, which led to a reduced number of grains per panicle and yield [22]. With an increase of nitrogen application rate, the effective panicle number, grain number per panicle, and yield increased, which was consistent with findings reported by Tian et al. [26]. Nitrogen is one of the basic elements of plant growth. Thus, increasing its application rate can promote the development of the rice root system and the accumulation of dry matter above the ground and enhance the nutritional structure and higher yield [26].

We found that the net photosynthetic rate of rice at high density was lower than that at low density, which was consistent with the finding of Xu et al. [6]. Increasing the transplanting density also increased the shading rate between rice leaves and degraded the ventilation conditions among rice populations [31,32], thus resulting in a decrease in net photosynthetic rate. In addition, the increased planting density also decreased the nitrogen content that accumulated per panicle, and the nitrogen content was positively correlated with photosynthetic ability [33]. Nitrogen application increased the net photosynthetic rate at low density, but the net photosynthetic rate did not increase with the increase of nitrogen application at high density, consistent with the findings reported by Hou et al. [23]. Nitrogen promoted chlorophyll synthesis in rice and increased the net photosynthetic rate of leaves [26]. However, high nitrogen input increased the nutritional structure of rice, ultimately increasing the shading rate and reducing the ventilation rate among plants [26] We found direct evidence that the photosynthetic rate at treatment $N_{1}$ was higher than at $\mathrm{N}_{2}$ at low planting density but not at high density.

In this study, increasing the transplanting density decreased the antioxidant enzyme activity of rice. There are few previous reports on the effect of transplanting density on antioxidant enzyme activity. However, Wu et al. showed that increasing the transplanting density would increase the risk of disease infecting rice and affect the redox system [33]. The risk of disease and insect pests caused by close planting may cause environmental stress on rice and alter the activity of antioxidant enzymes. High planting density reduced the carbohydrate accumulation per panicle, and a lack of nutrition may also be part of the reason for the decrease of antioxidant enzymes in rice. The antioxidant enzyme activity of treatment $\mathrm{N}_{2}$ was greater than that of $\mathrm{N}_{1}$ at low planting density, but opposite results were observed at a high planting density. Nitrogen application increased the activity of antioxidant enzymes, which was consistent with the conclusion of Zhong et al. [34]. Nitrogen can support the normal growth of plants and maintain the oxidation balance in plants $[35,36]$. In addition, some studies have found that nitrate consumption contributes to a higher ATP level in cells under stress, and the conversion of $\mathrm{NO}_{3}{ }^{-}$to $\mathrm{NH}_{4}{ }^{+}$is the main way to maintain a pH steady-state in plants under hypoxia [37]. This exhibited that nitrogen contributed to the normal oxidation balance in plants, reducing the production of free radicals and reactive oxygen species (ROS) as well as the consumption of antioxidant enzymes.

High density reduced PFPn, which was similar to findings reported by Lu et al. [38]. However, there were differences between our conclusions and those of that study, which may be because high planting density reduced rice yield (Table 2) and increased HIn, which was consistent with the conclusion of Xie et al. [27]. Increasing the planting density may accelerate the efficiency of $\mathrm{N}$ transport from stem and leaf to grain, and then increase the content of $\mathrm{N}$ in grain [27]. Increasing nitrogen application rates from level $\mathrm{N}_{1}$ to $\mathrm{N}_{2}$ decreased REn, AEn, and PFPn, which was consistent with the conclusion of Wang et al. [39]. This showed that there is a threshold for the nitrogen demand of plants, and excessive application of nitrogen fertilizer cannot be absorbed by plants, which may remain in the soil or be lost to the surrounding environment. Compared with treatment $\mathrm{N}_{0}$, only $\mathrm{N}_{1}$ increased HIn, which was consistent with the findings of Pan et al. [40]. These results 
indicated that increasing the nitrogen application rate reduced the transport of $\mathrm{N}$ from stem and leaf to the grain. High planting density decreased PFPn and HIn, proving that excessive nitrogen fertilizer was not absorbed or utilized by plants.

In the present study, we found that most tested traits were affected by environment, planting density, $\mathrm{N}$ application, and their interactions. Fortunately, the annual results showed consistent trends at different nitrogen applications and planting densities. To better understand the effects of $\mathrm{N}$ applications, planting density and their interaction on grain yield, nitrogen use efficiency, and antioxidant enzymes, it is necessary to further investigate these traits using more rice varieties across different ecological areas with diverse soils and climates.

\section{Conclusions}

We found that increasing planting density increased the effective panicle number but decreased the grain number per panicle, yield, net photosynthetic rate, antioxidant enzyme activity, PFPn, and HIn. Increasing the nitrogen application rate increased the effective panicle number, grain number per panicle, and yield, but this did not lead to an increase in net photosynthetic rate or antioxidant enzyme activity. REn, AEn, PFPn, and HIn decreased with the increase of nitrogen application rate. Most tested traits in this study showed significant differences over years, $\mathrm{N}$ applications, and between densities. The interactions between nitrogen fertilizer and transplanting density significantly affected the net photosynthetic rate, antioxidant enzyme activity, PFPn, and HIn. In general, increasing nitrogen application at low planting density increases the above indices, while increasing nitrogen application at high planting density does not have the same effect due to the waste of applied nitrogen.

Author Contributions: W.W. was the principal investigator who designed and implemented the research. C.S. and Q.X. oversaw the study and performed the statistical analysis. B.D. and S.Z. wrote the manuscript and provided guidance during experimentation. D.X. reviewed the final version of the manuscript before submission for peer review. All authors have read and agreed to the published version of the manuscript.

Funding: This research was funded by the Innovation and Application of Key Technologies for Light Simplified Direct Sowing of Rice in the North of the Middle and Lower Reaches of the Yangtze River (2017YFD0301403-06).

Data Availability Statement: Not applicable.

Acknowledgments: We thank Jianlong Xu (Beijing, China) for his critical comments on an earlier version of the manuscript.

Conflicts of Interest: The authors declare no competing interests.

\section{References}

1. Seck, P.A.; Diagne, A.; Mohanty, S.; Wopereis, M.C.S. Crops that feed the world 7: Rice. Food Secur. 2012, 4, 7-24. [CrossRef]

2. Banwart, S. Save our soils. Nature 2011, 7350, 151-152. [CrossRef] [PubMed]

3. Mueller, N.D.; Gerber, J.S.; Johnston, M.; Ray, D.K.; Ramankutty, N.; Foley, J.A. Closing yield gaps through nutrient and water management. Nature 2012, 490, 254-257. [CrossRef]

4. Tilman, D.; Balzer, C.; Hill, J.; Befort, L.B. Global food demand and the sustainable intensification of agriculture. Proc. Natl. Acad. Sci. USA 2011, 108, 20260-20264. [CrossRef] [PubMed]

5. Ye, Q.; Zhang, H.; Wei, H.; Zhang, Y.; Wang, B.; Xia, K.; Huo, Z.; Dai, Q.; Xu, K. Effects of nitrogen fertilizer on nitrogen use efficiency and yield of rice under different soil conditions. Front. Agric. China 2007, 1, 30-36. [CrossRef]

6. Xu, G.; Lu, D.; Wang, H.; Li, Y. Morphological and physiological traits of rice roots and their relationships to yield and nitrogen utilization as influenced by irrigation regime and nitrogen rate. Agric. Water Manag. 2018, 203, 385-394. [CrossRef]

7. Ju, C.; Buresh, R.J; Wang, Z.; Zhang, H.; Liu, L.; Yang, J.; Zhang, J. Root and shoot traits for rice varieties with higher grain yield and higher nitrogen use efficiency at lower nitrogen rates application. Field Crops Res. 2015, 175, 47-55. [CrossRef]

8. Ju, X.; Xing, G.; Chen, X.; Zhang, S.; Zhang, L.; Liu, X.; Cui, Z.; Yin, B.; Christie, P.; Zhu, Z. Reducing environmental risk by improving $N$ management in intensive Chinese agricultural systems. Proc. Natl. Acad. Sci. USA 2009, 106, 3041-3046. [CrossRef]

9. Li, P.; Lu, J.; Wang, Y.; Wang, S.; Hussain, S.; Ren, T.; Cong, R.; Li, X. Nitrogen losses, use efficiency, and productivity of early rice under controlled-release urea. Agric. Ecosyst. Eenviron. 2018, 251, 78-87. [CrossRef] 
10. Guo, J.H.; Liu, X.J.; Zhang, Y.; Shen, J.L.; Han, W.X.; Zhang, W.F.; Christie, P.; Goulding, K.W.; Vitousek, P.M.; Zhang, F.S. Significant acidification in major Chinese croplands. Science 2010, 327, 1008-1010. [CrossRef]

11. Liu, X.; Zhang, Y.; Han, W.; Tang, A.; Shen, J.; Cui, Z.; Vitousek, P.; Erisman, J.W.; Goulding, K.; Christie, P.; et al. Enhanced nitrogen deposition over China. Nature 2013, 494, 459-462. [CrossRef]

12. Yuan, S.; Nie, L.; Wang, F.; Huang, J.; Peng, S. Agronomic performance of inbred and hybrid rice cultivars under simplified and reduced-input practices. Field Crops Res. 2017, 210, 129-135. [CrossRef]

13. Hakeem, K.R.; Ahmad, A.; Iqbal, M.; Gucel, S.; Ozturk, M. Nitrogen-efficient rice cultivars can reduce nitrate pollution. Environ. Sci. Pollut. Res. 2011, 18, 1184-1193. [CrossRef] [PubMed]

14. Caine, R.S.; Yin, X.; Sloan, J.; Harrison, E.L.; Mohammed, U.; Fulton, T.; Biswal, A.K.; Dionora, J.; Chater, C.C.; Coe, R.A.; et al. Rice with reduced stomatal density conserves water and has improved drought tolerance under future climate conditions. New Phytol. 2019, 221, 371-384. [CrossRef]

15. Liu, Q.; Zhou, X.; Li, J.; Xin, C. Effects of seedling age and cultivation density on agronomic characteristics and grain yield of mechanically transplanted rice. Sci. Rep. 2017, 7, 14072. [CrossRef]

16. Berhanu, A.A. Effect of planting density on growth, yield and yield attributes of rice (Oryza sativa L.). Afr. J. Agric. Res. 2017, 12, 2713-2721. [CrossRef]

17. Huang, M.; Chen, J.; Cao, F.; Zou, Y. Increased hill density can compensate for yield loss from reduced nitrogen input in machine-transplanted double-cropped rice. Field Crops Res. 2018, 221, 333-338. [CrossRef]

18. Katsura, K.; Okami, M.; Mizunuma, H.; Kato, Y. Radiation use efficiency, N accumulation and biomass production of high-yielding rice in aerobic culture. Field Crops Res. 2010, 117, 81-89. [CrossRef]

19. Du, X.; Chen, B.; Shen, T.; Zhang, Y.; Zhou, Z. Effect of cropping system on radiation use efficiency in double-cropped wheatcotton. Field Crops Res. 2015, 170, 21-31. [CrossRef]

20. Zhao, X.; Zhou, N.; Lai, S.; Frei, M.; Wang, Y.; Yang, L. Elevated $\mathrm{CO}_{2}$ improves lodging resistance of rice by changing physicochemical properties of the basal internodes. Sci. Total Environ. 2019, 647, 223-231. [CrossRef]

21. Lu, G.; Casaretto, J.A.; Ying, S.; Mahmood, K.; Liu, F.; Bi, Y.; Rothstein, S.J. Overexpression of OsGATA12 regulates chlorophyll content, delays plant senescence and improves rice yield under high density planting. Plant Mol. Biol. 2017, 94, 215-227. [CrossRef] [PubMed]

22. Tang, L.; Gao, H.; Yoshihiro, H.; Koki, H.; Tetsuya, N.; Liu, T.; Tatsuhiko, S.; Xu, Z. Erect panicle super rice varieties enhance yield by harvest index advantages in high nitrogen and density conditions. J. Integr. Agric. 2017, 16, 1467-1473. [CrossRef]

23. Hou, W.; Khan, M.R.; Zhang, J.; Lu, J.; Ren, T.; Cong, R.; Li, X. Nitrogen rate and plant density interaction enhances radiation interception, yield and nitrogen use efficiency of mechanically transplanted rice. Agric. Ecosyst. Environ. 2019, 269, 183-192. [CrossRef]

24. Zhou, C.; Huang, Y.; Jia, B.; Wang, S.; Dou, F.; Samonte, S.O.P.; Chen, K.; Wang, Y. Optimization of Nitrogen Rate and Planting Density for Improving the Grain Yield of Different Rice Genotypes in Northeast China. Agronomy 2019, 9, 555. [CrossRef]

25. Liu, Y.; Li, C.; Fang, B.; Fang, Y.; Chen, K.; Zhang, Y.; Zhang, H. Potential for high yield with increased seedling density and decreased $\mathrm{N}$ fertilizer application under seedling-throwing rice cultivation. Sci. Rep. 2019, 9, 731. [CrossRef]

26. Tian, G.; Gao, L.; Kong, Y.; Hu, X.; Xie, K.; Zhang, R.; Ling, N.; Shen, Q.; Guo, S. Improving rice population productivity by reducing nitrogen rate and increasing plant density. PLoS ONE 2017, 12, e182310. [CrossRef]

27. Xie, X.; Shan, S.; Wang, Y.; Cao, F.; Chen, J.; Huang, M.; Zou, Y. Dense planting with reducing nitrogen rate increased grain yield and nitrogen use efficiency in two hybrid rice varieties across two light conditions. Field Crops Res. 2019, 236, 24-32. [CrossRef]

28. Zhu, X.; Zhang, J.; Zhang, Z.; Deng, A.; Zhang, W. Dense planting with less basal nitrogen fertilization might benefit rice cropping for high yield with less environmental impacts. Eur. J. Agron. 2016, 75, 50-59. [CrossRef]

29. Du, B.; Luo, H.; He, L.; Zheng, A.; Chen, Y.; Zhang, T.; Wang, Z.; Hu, L.; Tang, X. Deep fertilizer placement improves rice growth and yield in zero tillage. Appl. Ecol. Environ. Res. 2018, 16, 8045-8054. [CrossRef]

30. Gu, J.; Chen, Y.; Zhang, H.; Li, Z.; Zhou, Q.; Yu, C.; Kong, X.; Liu, L.; Wang, Z.; Yang, J. Canopy light and nitrogen distributions are related to grain yield and nitrogen use efficiency in rice. Field Crops Res. 2017, 206, 74-85. [CrossRef]

31. Lv, Y.; Xu, J.; Liu, X.; Wang, H. Vertical profile of photosynthetic light response within rice canopy. Int. J. Biometeorol. 2020, 64, 1699-1708. [CrossRef] [PubMed]

32. Keenan, T.F.; Niinemets, Ü. Global leaf trait estimates biased due to plasticity in the shade. Nat. Plants 2017, 3, 16201. [CrossRef]

33. Wu, W.; Wan, X.; Shah, F.; Fahad, S.; Huang, J. The Role of Antioxidant Enzymes in Adaptive Responses to Sheath Blight Infestation under Different Fertilization Rates and Hill Densities. Sci. World J. 2014, 2014, 502134. [CrossRef]

34. Zhong, C.; Cao, X.; Hu, J.; Zhu, L.; Zhang, J.; Huang, J.; Jin, Q. Nitrogen Metabolism in Adaptation of Photosynthesis to Water Stress in Rice Grown under Different Nitrogen Levels. Front. Plant Sci. 2017, 8, 1079. [CrossRef]

35. Chang, Z.; Liu, Y.; Dong, H.; Teng, K.; Han, L.; Zhang, X. Effects of Cytokinin and Nitrogen on Drought Tolerance of Creeping Bentgrass. PLOS ONE 2016, 11, e154005. [CrossRef]

36. Du, B.; Luo, H.; Liu, C.; Lei, C.; Jiang, S.; Lou, Y.; Xu, Y.; Wang, M.; Shi, L.; Xing, D. Effects of different water management methods on seeding rate, phenological and yielding properties of different rice cultivars (Oryza sativa L.). Appl. Ecol. Environ. Res. 2019, 17, 4269-4279. [CrossRef] 
37. Vijayan, J.; Senapati, S.; Ray, S.; Chakraborty, K.; Molla, K.A.; Basak, N.; Pradhan, B.; Yeasmin, L.; Chattopadhyay, K.; Sarkar, R.K. Transcriptomic and physiological studies identify cues for germination stage oxygen deficiency tolerance in rice. Environ. Exp. Bot. 2018, 147, 234-248. [CrossRef]

38. Lu, J.; Wang, D.; Liu, K.; Chu, G.; Huang, L.; Tian, X.; Zhang, Y. Inbred varieties outperformed hybrid rice varieties under dense planting with reducing nitrogen. Sci. Rep. 2020, 10, 8769. [CrossRef]

39. Wang, Z.; Zhang, W.; Beebout, S.S.; Zhang, H.; Liu, L.; Yang, J.; Zhang, J. Grain yield, water and nitrogen use efficiencies of rice as influenced by irrigation regimes and their interaction with nitrogen rates. Field Crops Res. 2016, 193, 54-69. [CrossRef]

40. Pan, J.; Liu, Y.; Zhong, X.; Lampayan, R.M.; Singleton, G.R.; Huang, N.; Liang, K.; Peng, B.; Tian, K. Grain yield, water productivity and nitrogen use efficiency of rice under different water management and fertilizer-N inputs in South China. Agric. Water Manag. 2017, 184, 191-200. [CrossRef] 